\title{
The Law of State on Religious Communities during Zogu's Governance and Enver Hoxha's Governance (The Example of the Catholic Church in Albania)
}

\author{
Prof.Assoc. Nertila Haxhia Ljarja \\ University of Shkodra "Luigj Gurakuqi" \\ Department of History
}

Doi:10.5901/jesr.2014.v4n4p426

\begin{abstract}
This essay is a historical analysis which compares the common cause as well as differing viewpoints from the people with regards to the Catholic Church. The main focus is on the law that required it to be under complete control of the state. Both time periods that have been analysed below, had the ultimate aim of separating the state from religion. The varying political views that were followed managed to alienate the relationship with the Catholic Church in Albania, this was especially the case during times of Communism.
\end{abstract}

Keywords: Catholic Church in Albania, religion, monarchic state, dictatorial state.

\section{II Trattamento Legislativo delle Comunità Religiose nello Stato Monarchico di re Zog e Quello Dittatoriale di Enver Hoxha (II Caso della Chiesa Cattolica in Albania)}

\subsection{Decreto - legge sulla formazione di comunità religiose nel periodo della monarchia Zogista}

II $1^{\circ}$ settembre 1928 l'Assemblea decise all'unanimità la proclamazione dello Stato Albanese come monarchia, proclamando Ahmet Zog il Re degli albanesi. Immediatamente iniziò il lavoro per la modifica dei precedenti Codici come quello penale, civile e commerciale, basandosi sul modello europeo. Inoltre accelerarono anche le preparazioni per la legge sulle comunità religiose, approvata il 16 luglio 19291, in base alla quale gli venivano consentite il riconoscimento della personalità giuridica e l'approvazione dello statuto riguardo al loro funzionamento. In base all'articolo 1 della suddetta legge, chiunque gode della libertà di coscienza e di religione che desidera, libertà questa garantita dagli articoli 5 e 203 dello Statuto Fondamentale del Regno. Attraverso questa legge il re Zog intendeva ottenere il pieno controllo delle tre comunità religiose ${ }^{2}$. Inoltre il prossimo obiettivo era la sottomissione della popolazione cattolica, con centro la città di Scutari, dove operavano istituzioni educative e culturali importanti come il Convento Pontificio, il Collegio Saverino, la tipografia gesuita ecc. Secondo il ricercatore Della Rocca: " Zog, appena salito al potere, alla fine del 1922, prende misure a danno dei cattolici albanesi i quali generalmente non sono suoi sostenitori e hanno nelle loro fila alcuni dei suoi avversari decisi."

La politica di Zog è stata formalemente indirizzata verso una laicità assoluta, (non causata dai bisogni pratici della modernizzazione dello Stato, ma dal suo desiderio di limitare i poteri politici dei potenziali avversari, interni ed esterni), e da un punto di vista pratico intesa a sottomettere le comunità religiose all'autorità dello Stato.

Nel decreto di Re Zog del 16 luglio 1929 viene sancito ufficialmente l'esistenza di tre religioni in Albania, che in un breve periodo dovrebbe elaborare i propri statuti. Secondo questo decreto, le comunità religiose non avrebbero più l'appartenenza giuridica all'interno dello Stato Albanese, dovrebbero essere riconosciute dallo Stato, il quale decide sulla nomina degli amministratori più alti e controlla la loro attività finanziaria. Le comunità religiose, secondo il decreto, devono astenersi da qualsiasi attività politica, non devono ricevere sovvenzioni dall'esterno e non devono avere relazioni con istituzioni, organizzazioni e persone straniere, oltre a legami culturali e spirituali. Tramite questo decreto le comunità religiose venivano sottomesse all'autorità statale, fatto inaccettabile per loro.

\footnotetext{
${ }^{1}$ Art. 1 "Çdo njeri ne Shqipni gëzon lirinë e ndërgjegjes dhe të fesë që dishron; kjo liri është e garantueme me artikujt 5 e 203 të statutit themeltar të Mbretnisë." Dekret-Ligjë mbi Formimin e Komuniteteve Fetare, 9 korrik 1929. Fletore Zyrtare Nr. 38 datë 16 Korrik 1929.

2 "Sikundër shkollat, Zogu donte t'i lidhte më fort pas shtetit edhe bashkësitë fetare dhe t'i clironte ato nga ndikimet e huaja. Që në korrik 1929 atyre iu kërkua të hartonin statute dhe t'ia paraqisnin ato qeverisë për shqyrtim. Nga ana tjetër bashkësitë u ndaluan të marrin ndihma financiare nga jashtë vendit. Drejtuesit e bashkësive fetare duhej të miratoheshin nga mbreti". Peter Bartl, Shqiptarët, Instituti i Dialogut \& Komunikimit, Tiranë, pg.212.

${ }^{3}$ Roberto Morocco della Rocca, Kombësia dhe feja në Shqipëri 1920-1944, Shtëpia Botuese "Elena Gjika", Tiranë 1994. pg.84.
} 
Nello Statuto Fondamentale del Regno albanese veniva citato: "Lo Stato albanese non ha una religione ufficiale. Tutte le religioni e credenze sono considerate sacre e la loro esterna libertà di pratica è garantita. La religione non può formare ostacoli legali, in qualsiasi modo. Religioni e credenze non possono eventualmente essere utilizzati per scopi politici". ( Art.5 ). Questa legge esprime chiaramente la politica religiosa che avrebbe successivamente seguito lo Stato albanese verso la laicità, la libertà e l'uguaglianza religiosa, verso una completa sottomissione delle comunità religiose.

La richiesta per la redazione dello statuto non ha preso risposta dalla Chiesa cattolica che non può accettare una Chiesa cattolica nazionale formata solo da clero autoctono, chiuso ai missionari stranieri, soggetto al controllo del governo, un punto questo che di vista dogmatico è stato completamente inaccettabile. Qualcosa di simile verrà eseguito dopo l'arrivo al potere dei comunisti. La Santa Sede aveva molto paura che le misure del 1929, avrebbero portato ad una vera e propria resa dei conti. Questo dubbio derivava dal fatto che il decreto statale era stato in contrasto con alcuni principi dogmatici, ai quali la Chiesa romana non poteva rinunciare. A questo punto, il Vaticano esercitò pressioni sul governo italiano per intervenire sul governo di Tirana.

Nel nuovo Codice civile (redatto dal modello francese), una serie di articoli erano in contrasto con i principi fondamentali del diritto canonico, come probabilità prevista di divorzio. Dopo la promulgazione del codice civile, la famiglia non sarebbe più l'oggetto del diritto canonico. ${ }^{4} \mathrm{Ma}$ comunque Zog era determinato a non fare concessioni su questo punto, perché questo porterebbe la sottomissione del diritto civile a norme ecclesiastiche.

Ma il più grave conflitto tra il governo e la Chiesa avvenne riguardo alle scuole cattoliche, fatte chiudere nel $1933^{5}$ per riaprire il 9 maggio 1936. La presenza cattolica nel campo educativo, soprattutto nel nord dell'Albania, è un fattore importante anche socialmente. II divieto di istruzione privata era collegato con l'elemento cattolico in questo settore. Forse questo era uno dei motivi che chiudendo tutte le scuole private straniere, lo scopo era di limitare la diffusione delle scuole finanziate dall'Italia (nei casi in cui Zog in questo periodo cercava di staccarsi un po' dalla dipendenza italiana, questa è stata una misura sopratutto anti italiana) che specificamente era incentrata sulla popolazione cattolica del nord. ${ }^{6}$ I fattori che hanno contribuito a risolvere il problema sono stati accordi politici con gli italiani nel 1936, in cui uno degli elementi che è stato rivisto era la questione educativa e la frase di Zog dal Consiglio della Società di Nazioni Unite, che nel 6 aprile 1935 criticava la politica educativa del Regno albanese.

II 20 maggio 1933, Monsignor Lazër Mjeda, con la qualità del primato della Chiesa e arcivescovo di Scutari, Monsignor Pjetër Gjura, arcivescovo di Durazzo , Monsignor Benardin Shllaku, vescovo di Pult, Monsignor Luigj Bumçi, vescovo di Lezha, Mons. Zef Gjonali vescovo di Sapa, Mons. Frano Mirdita, vescovo abate di Mirdita, hanno inviato una petizione alla Corte Permanente di Giustizia Internazionale presso il Consiglio Supremo della Società di Nazioni Unite a Ginevra, in cui trattavano le condizioni della comunità cattolica nel Regno albanese. ${ }^{7}$

Tuttavia dobbiamo sottolineare che i cambiamenti di atteggiamento non si aspettavano nemmeno dal Vaticano, né dai membri della Chiesa cattolica in Albania, ma soprattutto da parte dello Stato albanese. In un lento processo "di obbedienza", a quanto pare, finalmente, il governo albanese decise di ritirarsi.

Le scuole cattoliche nel Settembre del1936 vennero riaperte perché la loro chiusura, in qualche modo, si prosegue lungo un precedente molto pericoloso per la stabilità socio - politica in Albania. Questa politica perseguita dai governanti del regno manifestava l'esistenza di una disposizione ufficiale contro la libertà attraverso l'uso improprio del processo di laicizzazione, una disposizione segreta antieuropea e anti- cattolica, dissimulata attraverso l'azione di alcuni individui

\footnotetext{
${ }^{4}$ Giovanni Cimbalo, Pluralizmi i besimit dhe komunitetet fetare në Shqipëri, Naimi, Tiranë, 2013, pg.72-74.

5 “...Në prill 1933 u mbyllën të gjitha shkollat private. Nga kjo masë nuk ishin të prekura vetëm shkollat italiane, por edhe shkollat e bashkësive fetare, përderisa nuk shërbenin vetëm për shkollim të klerikëve.... Nga fundi i vitit 1935 minoriteteve sërish u njihet e drejta, për të mbajtur shkolla private. Më 1936 ndalimi i shkollave private u pezullua krejtësisht. ... Katolikët kishin pjesëmarrje të madhe në lëvizjen kombëtare dhe falë organizimit të mirë dhe nivelit arsimor të priftërinjve kisha katolike luante edhe më tej rol shumë më të rëndësishëm në jetën kulturore e shoqërore të vendit, se ç'mund të supozohet nga përqindja e vogël e katolikëve në popullsinë e gjithëmbarshme. Raporti në mes të mbretit mysliman dhe katolikëve, të cilët në qeveri merrnin pjesë shumë rrallë, nuk ishte i patrazuar, sidomos pasi që më 1933 ishin mbyllur shkollat e jezuitëve dhe françeskanëve në Shkodër. Por të dy palët përpiqeshin të afroheshin, meqë kishin interesa të përbashkëta: kishës katolike $i$ duhej për shkollat e veta mbështetja financiare nga shteti, ndërsa Zogut $i$ duhej kisha për planet e veta martesore. Nusja duhej të ishte nga një familje fisnike europiane dhe mund të ishte katolike." Peter Bartl, Shqipëria. Nga mesjeta deri sot. Drita, Prizren 1999, pg.200-202.

6 "Nacionalizimi i sistemit arsimor kishte për qëllim t'i vinte ledh përhapjes së shkollave të sponsorizuara drejtpërdrejt nga Qeveria italiane për katolikët e Veriut.... Kështu që qeveria u përpoq të eliminonte propagandën shkombëtarizuese, që ishte e pashmangshme në shkollat e agjencive fetare të huaja dhe përçarëse,., Edwin Jacques, Shqiptarët. Historia e popullit shqiptar nga lashtësia deri ne ditët e sotme. Fondacioni Abraham Linkoln "Kartë e Pendë", Tiranë., pg.432-433.

${ }_{7}$ PRO. FO. 371. 16625, C 8029/8029/90, Petition of the Catholic Archbishops and Bishops in Albania. Vedi anche: Roberto Morocco della Rocca, Kombësia dhe feja në Shqipëri 1920-1944, Shtëpia Botuese "Elena Gjika”, Tiranë 1994. pg.142-169.
} 
governanti cattolici incorporati nelle loro dottrine anti-clericali, o facilmente gestibili. Tale politica sarebbe stata un indicatore importante che prefigurava le difficoltà che avrebbe affrontato il paese nel prossimo secolo, nell'avanzamento verso la democrazia, lo stato di diritto e l'occidentalizzazione. Inoltre, fu un indicatore del fatto che l'equilibrio nella direzione politica del paese si sarebbe ulteriormente polarizzato da potenti forze conservatrici e non dalla parte più emancipata, illuminata e civile di questo paese.

Quello dei cattolici era un caso specifico, perché nei loro confronti, il re non rispettò pienamente la legge sulle comunità religiose. Questa legge stabiliva l'obbligo che il clero attivo in Albania avrebbe dovuto essere di nazionalità albanese ed è stato previsto il controllo statale sulla gestione della tenuta di ogni religione. ${ }^{8} \mathrm{Ma}$ in pratica l'applicazione della legge non era la stessa per tutte le comunità religiose albanesi. I cattolici, contrariamente ai mussulmani e ortodossi, rifiutarono di sottoporsi allo Stato, ai sensi della legge del 16 luglio 1929 per la creazione di comunità religiose ufficialmente riconosciute. Come risultato, hanno rinunciato al profitto della personalità giuridica, dal diritto di essere assistiti economicamente dallo stato, hanno continuato i legami non solo spirituali, ma anche amministrativi e finanziari con la Santa Sede (che è stato interamente conforme alla legittimità di fede dei membri della chiesa universale) ed elementi di clero cattolico non albanesi hanno continuato l'attività in Albania.

I cattolici non sono stati sottoposti alla legge del luglio 1929 creando così un clima di incertezza giuridica nelle relazioni tra lo Stato e la Chiesa cattolica che continuò fino al $1939 .{ }^{9}$

Quasi lo stesso percorso sarà seguito dai comunisti albanesi, con l'arrivo al potere, che ripeteranno in campo educativo le stesse politiche impressionanti contro il clero, ma questa volta con conseguenze fatali per il futuro delle giovani generazioni. Per raggiungere questo obiettivo avranno la storia degli anni 1933-1936, come punto importante di riferimento.

\subsection{Decreto "Sulle comunità religiose" nel periodo della dittatura comunista}

Tra le prime misure intraprese della dittatura comunista contro le comunità religiose in Albania è stata la realizzazione della riforma agraria attraverso la confisca dei loro beni. Particolarmente grave colpo subì la Chiesa cattolica come in possesso di molteplici attività. Particolarità per la Chiesa cattolica è stato il fatto che da quando ha rinunciato alla personalità giuridica civile in legislazione di Zog, gli fu affidato le sue possessioni ai terzi, e il governo ha reso più facile implementare su questi individui le misure di austerità in temi di proprietà. Lo stato manteneva uno stretto controllo su tutto attraverso il sostegno del clero con i stipendi. "La sopravvivenza delle comunità religiose si condizionava sugli aiuti dello Stato. Nel corso del tempo, la perdita di indipendenza economica spinge le comunità religiose verso la bancarotta economica, come uno dei mezzi più efficaci per la loro distruzione e per soddisfare la strategia a lungo termine volta a eliminare la religione in Albania." ${ }^{10}$ Oltre al collasso economico, la Chiesa cattolica già soffriva una crisi delle sue strutture come risultato di allontanamento dal paese del clero con cittadinanza straniera e la condanna di molti altri a lunghe pene detentive e in molti casi le condanne a morte.

Possiamo dire che, in termini di misure economiche relative alla confisca del patrimonio delle istituzioni religiose, questo era un fenomeno nuovo che non si incontra nei periodi precedenti o periodi analizzati sopra.

Un'altra area di confronto tra lo Stato e la Chiesa cattolica era quella dell'educazione attraverso la riforma dell'istruzione che ha avuto due direttrici principali: la secolarizzazione dell'insegnamento e la separazione della scuola dalla religione. Questa riforma ha raggiunto il suo picco nel 1947, quando una circolare del Ministero della Pubblica Istruzione ha anche vietato l'insegnamento della religione nelle scuole pubbliche. Queste misure hanno portato al divieto di insegnamento e di preparazione dei prossimi clerici e anche la chiusura della stampa religiosa. Passo dopo passo si viene riducendo lo spazio delle comunità religiose e crescendo il controllo statale su di loro.

II 26 novembre 1949, il governo comunista annunciò il decreto nr. 743 intitolato "Sulle comunità religiose"11, attraverso la quale era vietata l'attività religiosa di culto nelle moschee e nelle chiese e li limitano solo a tenere servizi religiosi. Questo decreto limitava il diritto dei credenti a seguire le pratiche religiose e di praticare le loro credenze religiose, a condizione che tali norme e credenze religiose non dovrebbero essere in contrasto con le "leggi dello stato, la legge e l'ordine, e di buoni costumi". II decreto ha inoltre richiesto che tutte le lettere pastorali e altre comunicazione di

${ }^{8}$ PRO.FO.371.8532 pg.84. Vedi anche: Statuti themeltar i Mbretnisë Shqiptare. In "Titulli l": "Shteti Shqiptar nuk ka fe zyrtare. Të gjitha fetë dhe besimet janë të nderueme dhe liria e ushtrimit dhe e praktikimit të jashtme të tyre asht sigurue. Feja nuk mund të formojë pengime juridike me asnjë mënyrë. Fetë dhe besimet kurrsesi nuk mund të përdoren për qëllime politike".

${ }_{9}^{9}$ Roberto Morocco della Rocca, Kombësia dhe feja në Shqipëri 1920-1944, Shtëpia Botuese "Elena Gjika", Tiranë 1994. pg.141-144.

10 Giovanni Cimbalo, Pluralizmi i besimit dhe komunitetet fetare në Shqipëri, Naimi, Tiranë, 2013, pg.108.

${ }^{11}$ AQSH. F.890, v.1951, D.561, fl.2. 
comunità religiose, dovrebbero prendere previa approvazione dal parte delle autorità statali 0 dovrebbero essere considerati prima dai funzionari locali del Partito. Secondo questo decreto, le organizzazioni religiose devono servire ed essere fedeli allo Stato, che deciderà sul sostegno finanziario di chiese e moschee. Vietato i rapporti del clero con "altra classe all'estero". 12

Alla fine, il decreto ordinava ciascuno dei quattro principali comunità religiose in Albania, il musulmano, bektashi, ortodossi e cattolici, per preparare i loro progetti di statuti e di presentarla entro 90 giorni ai organi dello Stato. Nel progetto di statuto va sottolineato fedeltà al governo. Dato che nessuna delle comunità religiose non può portare una cosa del genere in questo breve periodo di tempo interamente ad una svolta così radicale, i statuti di ogni comunità sono stati dettati dal Consiglio dei Ministri della Repubblica Popolare d'Albania e immessi sul loro conto. ${ }^{13}$

II problema della separazione tra la Chiesa e la Santa Sede e il papato, verrà inserito in questo periodo al centro dello scontro tra lo Stato e la Chiesa, o più precisamente: il resto della Chiesa cattolica in Albania. Ma la Chiesa non poteva fare concessioni, perché questo porterebbe a una chiesa autocefala, il che è inaccettabile per il cattolicesimo perché era contraria alle leggi dogmatiche e il funzionamento amministrativo della chiesa nel suo complesso.

Nel 1950, i leader della dittatura comunista, aveva iniziato qualche sforzo per ottenere il sostegno nel paese e attirare elemento non - comunista. In questo contesto, hanno cominciato un "approccio" con il clero. L'élite della Chiesa era quasi tutto finito, e non c'era alcuna possibilità di sostituirli dopo che i seminari gesuiti e francescani furono completamente eliminati. La necessità di questo dialogo in questi momenti viene dal fatto che lo Stato è stato costretto davanti agli internazionali, come facciata, per costruire relazioni formali con il clero cattolico. I sacerdoti sono stati annunciati che inizierà la discussione di un "progetto" di una legge, che avrebbe dovuto essere firmato dal clero, per ordine dello Stato. Dal momento che i musulmani ed i cristiani ortodossi non avevano problemi significativi, loro avevano approvato i loro statuti. Mentre per i cattolici lo scontro importante era collegato con la Santa Sede (Vaticano), e l'incapacità di un distacco dal Papa. Ora, con le fucilazioni e la scomparsa delle figure chiave principali del clero, si sperava che si sarebbe mosso qualcosa.

Nel decreto $\mathrm{nr} .743$, si ordinavano tutte le comunità religiose a presentare un statuto, che dovrebbe essere in armonia con la costituzione vigente..$^{14}$ Lo statuto sarà analizzata dal governo per l'approvazione. Questo decreto divenne la legge "Sulle comunità religiose", Nr.743, dt.26.11.1949. Questa legge cercava da tutti gli organismi religiosi di dichiarare la loro fedeltà al Partito e il Governo albanese. Altre comunità erano soggette a questo ordine ${ }^{15}$, ma solo la Chiesa cattolica creava problemi.

La legge era destinata a fissare di nuovo le relazioni tra le comunità religiose e lo Stato. Non si prevedeva solo la questione dello statuto, ma la legge costringeva le comunità religiose a cercare dai credenti "la fedeltà al governo popolare e della Repubblica Popolare d'Albania" per rafforzare l'identità nazionale. I leader delle comunità religiose che portavano i "contatti canonici" con l'esterno, devono essere certificati da parte del Consiglio dei Ministri - questo articolo trattava solo con la Chiesa cattolica in Albania. I Statuti ufficiali delle altre sette religiose sono stati elaborati e approvati dal governo. Solo quello della Chiesa cattolica è tornato tre volte. ${ }^{16}$ L'atteggiamento severo del governo nei confronti

${ }^{12}$ AQSH. F.890, v.1951, D.561, fl.3.

${ }^{13}$ Owen Pearson, Albania in the twentieth century:A history, volume three, Albania as Dictatorship and democracy, From Isolation to the Kosovo War, London 2006, pg. 331.

14 "Perspektiva e zhvillimit të vendit tonë diktonte nevojën e përpunimit të një vije politike të drejtë dhe e një qëndrimi të përshtatshëm ndaj aparatit fetar dhe ideologjisë fetare. Thelbi $i$ këtij qëndrimi duhej të ishte $i$ tillë që t'i shërbente vetëm shpejtimit të "vdekjes së natyrshme" të ideologjisë fetare. Vetëm në këtë drejtim do të përpunohej dhe do të kalonte në provë vija politike e partisë dhe e shtetit tonë për sa i përket problemit të fesë. Në këtë drejtim vepronin krejt masat dhe reformat socialo-ekonomike me karakter demokratik apo socialist, por më drejtpërdrejt vepronin masa e parime të tilla siç ishin: Ndarja e komuniteteve fetare nga shteti dhe nga qendrat fetare të huaja. Ndarja e shkollës nga feja dhe laicizimi i saj. Shpallja e fesë si "çështje private" e shtetasve." Hulusi Hako, Gjyq Zotit, Shtëpia botuese e librit politik, Tiranë 1972, pg.20.

${ }^{15}$ Nicholas Pano, "Feja në Shqipëri: trashëgimia e epokës komuniste", Fetë dhe Civilizimet në Mijëvjeçarin e ri - Rasti i Shqipërisë, Konferenca Ndërkombëtare, Tiranë, 14-15 nëntor 2003, pg.150.

16“Në mbledhjen e tij të datës 22 dhjetor 1950, Sekretariati i Komitetit Qendror të Partisë së Punës të Shqipërisë ...ndër të tjera u diskutua edhe çështja e statutit të klerit katolik; pasi dëgjoi raportin e shokut Mehmet Shehu mbi gjithë këto çëshjte, Sekretariati konstatoi: Projektstatuti i tretë i klerit katolik bie në kundërshtim me Ligjin mbi komunitetet fetare, në nenin 13 të tij ku, sipas propozimit të klerit katolik, hierarkët e Kishës Katolike (Vikarët, Kapitullarët dhe Metropoliti) zgjidhen nga Konferenca e klerit katolik, i parashtrohen Papës për konfirmim dhe pastaj i paraqiten qeverisë së Republikës Popullore të Shqipërisë, për aprovim; kurse sipas ligjit të komuniteteve fetare, këta hierarkë, pasi të zgjidhen nga Konferenca e klerit katolik, i paraqiten qeverisë për aprovim e, pastaj mund të kërkojnë në mënyrë shtetërore bekimin e Papës. Nenet e tjera të projektstatutit, pothuajse të gjitha pajtohen plotësisht me Ligjin mbi komunitetet fetare. Për të zgjidhur çështjen e përmbaitjes është kërkuar mendimi, në formë këshillimi, i shokëve sovjetik... Ndërsa barrën kryesore në këtë pikë, duhet ta mbante Sigurimi i Shtetit, i cili duhej të shfrytëzonte disa mosmarrëveshje që kishin drejtuesit e 
della chiesa era ispirato anche dall'accettazione ragionevole del statuto di governo d'altre sette religiose. Poi, dopo molti scontri, il governo ha interrotto i colloqui con la chiesa e riprese la sua politica di intimidazione con la violenza. Lanciò nuove misure per arrestare i sacerdoti, specialmente quelli che stavano mostrando più resistenza. Nonostante questa ripetuta pressione, il clero rimase unito. ${ }^{17}$

Nel 26 giugno 1951 i clerici cattolici sopravvissuti dal genocidio di cinque anni prima, sotto forte pressione da parte dei promotori di questo genocidio, nella loro assemblea generale, approvarono il nuovo statuto. ${ }^{18}$ La formazione della Chiesa Cattolica Nazionale Albanese "ispirata a principi religiosi della Chiesa Universale Cattolica, che è stata fondata da Gesù Cristo a capo Papa, successore di Pietro", ma senza tenere "con il Papa alcun legame organizzativo, economico e politico", è stata la legge firmata ${ }^{19}$ dall'unico vescovo sopravvissuto e dai 63 sacerdoti religiosi e secolari, di cui 2 da altre fonti figuravano morti e 10 prigionieri, mentre quattro sacerdoti gesuiti figuravano come sacerdoti secolari. ${ }^{20}$

II 30 giugno 1951 il Governo ha approvato il nuovo Statuto della Chiesa Cattolica Albanese, presumibilmente "sulla base della decisione presa dall'assemblea generale degli ecclesiastici albanesi a Scutari nel 26 giugno 1951". II decreto è stato etichettato come "Approvazione dello Statuto della Chiesa Cattolica d'Albania ", e lo statuto è diventato legge. La legge ha dichiarato che la Chiesa Cattolica Albanese non doveva avere legami organizzativi, politici o economici con il Vaticano e che il coordinamento degli affari religiosi deve assolutamente passare solo attraverso i canali statali. ${ }^{21}$

La legge prevedeva una completa sottomissione allo Stato, e bisogna notare che, alcuni articoli (divieto di partecipazione in politica, i statuti, il sostegno del patriottismo) erano anche in un decreto dello stesso nome di Re Zog,

komunitetit katolik të asaj kohe, për të” izoluar" elementët reaksionarë. Në lidhje me të gjitha këto, pasi e diskutoi çdo çështje veçmas, Sekretariati i Partisë së Punës së Shqipërisë, vendosi: Për të zgjidhur çështjen e nenit 13 të projektstatutit të tretë të Kishës Katolike, të pritet mendimi i shokëve sovjetikë! Ndërkohë, të punohet nga ana e organeve të Partisë dhe Sigurimit të Shtetit për të thelluar e për të shfrytëzuar kontradiktat e brendshme të klerit katolik, me qëllim që të izolohen elementët reaksionarë e të binden masat katolike, si dhe klerikët e vegjël e të mesëm, për drejtësinë e politikës së shtetit në lidhje me Kishën Katolike!". Citato da Pjetër Pepa, Tragjedia dhe lavdia e Klerit Katolik në Shqipëri, Shtëpia Botuese 55, Tiranë 2007, Vell.Il, pg.218-220.

${ }^{17}$ AQSH. F.131, v.1951, D.7, fl.1.

${ }^{18}$ Ajet Shahu, "Shteti komunist dhe Kisha Katolike (1945-1967)". Krishtërimi ndër shqiptar, Simpozium Ndërkombëtar, Tiranë, 16-19 Nëntor 1999. Toena, Tiranë 2000, pg. 355-367. Vedi anche: Owen Pearson, Albania in the twentieth century:A history, volume three, Albania as Dictatorship and democracy, From Isolation to the Kosovo War, London 2006, pg. 428 "A general assembly of the Roman Catholic Clergy was convened in Shkodër at which Monsignor Bernardin Shllaku, Bishop of Pulati, presented the draft of a proposed new charter of the Albanian Catholic Church which he had received in Tirana from Mehmet Shehu who had personally beaten him. Under this charter a "national" Catholic Church would have no jurisdictional, economic, or political relations with Vatican. The task of the clergy would be "to develop feelings of devotion to the people's will". Canon Law would apply only when it did not conflict with the laws of the Albanian Republic, to which the Church would submit. The government would subsidize the Church in accordance with needs and the State's resources. In the course of the deliberations Bishop Shllaku was asked to clarify the meaning of the first paragraph of the charter which stated: "The Church does not have any organizational, economic, or poltical relation with Pope". Bishop Shllaku answered that Mehmet Shehu had informed him that the Albanian Govermnet expected the Albanian Catholic Church to be removed from the political sphere of the Vatican, but that it did not exclude spiritual relationship. The Metropolitan Archbishop might communicate with religious communities abroad, but such messages might deal with religious questions only and must pass thruogh officials of the Albanian government. After continued harassment and pressure from the government, tentative agreement was reached at the ende of the painful discussions and the charter was signed, in the hope that the government would be liberal and sincere in honouring its verbal and written guarantees. There was also the fear that, if the charter was not signed, persecution would be renewed, and the structure of the Church in Albania, already so weakened, would be completely collapse. It was agreed that the most important and fundamental factor, the unity within the Church, was saved and spiritual ties with the Hole See were kept."

${ }^{19} 64$ është numri i meshtarëve që me datë 26.6.1951 firmosën Statutin e Kishës Katolike të Shqipërisë, në mbledhjen e përgjithshme të Klerit katolik shqiptar, nën kryesinë e Imzot Bernardin Shllakut. Në këtë dokument mungojnë firmat e Dom Marjan Artës, Dom Nikoll Gjinajt, Dom Ejëll Gurakuqit, Padër Benedikt Demësetj., të cilët megjithëse figurojnë në fotot e bëra me këtë rast , janë arrestuar apo internuar, ose nuk kanë marrë pjesë për arsye të tjera në aktin final të miratimit. Gjithashtu mungojnë firmat e Padër Ferdinand Pali, Dom Zef Gilaj, Padër Viktor Volaj, Dom Ndre Lufi etj që kanë qënë në burg ose internim dhe i një grupi tjetër që s'mori pjesë me dashje, në formë proteste, në firmosjen e statutit si, Dom Ejëll Kovaçi, Dom Dedë Malaj. Padër Konrad Gjolaj etj që shumë shpejt do vuajnë tragjikisht për këtë veprim. Pjetër Pepa, Tragjedia dhe lavdia e Klerit Katolik në Shqipëri, Shtëpia Botuese 55, Tiranë 2007, Vell.Il, pg.243.

20 Atë Zef Valentini, Ernest Koliqi, Relazione della Chiesa perseguitata d'Albania, (Biblioteca della Chiesa Francescana, Shkodër), pg.12, manoscritto.

${ }^{21}$ Owen Pearson, Albania in the twentieth century:A history, volume three, Albania as Dictatorship and democracy, From Isolation to the Kosovo War, London 2006, pg. 430. Ulteriormente nel diario si legge: "The church leaders, under Bishop Bernardin Shllaku, were forced to accept the nationalization of religious communities, but they remained adamant that, even of organizational and economic relations with Rome had to be cut, in no way would they renounce the spiritual sovereignity of the Pope or their allegiance to Jesus Christ, the founder of their Church". 
del 16 giugno 1929.

\section{Bibliografia}

AQSH PRO. FO.

Statuti themeltar i Mbretnisë Shqiptare

Fletore Zyrtare Nr. 38 datë 16 Korrik 1929.

Manoscritto:

Atë Zef Valentini, Ernest Koliqi, Relazione della Chiesa perseguitata d'Albania, (Biblioteka e Kishës Françeskane, Shkodër).

Fetë dhe Civilizimet në Mijëvjeçarin e ri - Rasti i Shqipërisë, Konferenca Ndërkombëtare, Tiranë, 14-15 nëntor 2003.

Krishtërimi ndër shqiptar, Simpozium Ndërkombëtar, Tiranë, 16-19 Nëntor 1999. Toena, Tiranë 2000.

Edwin Jacques, Shqiptarët. Historia e popullit shqiptar nga lashtësia deri ne ditët e sotme. Fondacioni Abraham Linkoln "Kartë e Pendë", Tiranë.

Giovanni Cimbalo, Pluralizmi i besimit dhe komunitetet fetare në Shqipëri, Naimi, Tiranë, 2013.

Hulusi Hako, Gjyq Zotit, Shtëpia botuese e librit politik, Tiranë 1972.

Owen Pearson, Albania in the twentieth century:A history, volume three, Albania as Dictatorship and democracy, From Isolation to the Kosovo War, London 2006.

Peter Bartl, Shqiptarët, Instituti i Dialogut \& Komunikimit, Tiranë.

Pjetër Pepa, Tragjedia dhe lavdia e Klerit Katolik në Shqipëri, Vell.II, Shtëpia Botuese 55, Tiranë 2007.

Roberto Morocco della Rocca, Kombësia dhe feja në Shqipëri 1920-1944, Shtëpia Botuese "Elena Gjika", Tiranë 1994. 